\title{
Thermal charm production by massive gluons and quarks
}

\author{
Péter Lévai ${ }^{1,2}$ and Ramona Vogt ${ }^{2,3,4, \star}$ \\ ${ }^{1}$ KFKI Research Institute for Particle and Nuclear Physics, \\ POB. 49, Budapest, 1525, Hungary \\ ${ }^{2}$ Institute for Nuclear Theory, University of Washington, \\ Seattle, WA 98195 \\ ${ }^{3}$ Nuclear Science Division, Lawrence Berkeley National Laboratory, \\ Berkeley, CA 94720 \\ ${ }^{4}$ Department of Physics, UC Davis, Davis, CA 95616
}

October 10, 2018

\begin{abstract}
We investigate charm production in an equilibrated quark-gluon plasma produced in heavy-ion collisions at RHIC and LHC. Effective quark and gluon masses are introduced from thermal QCD calculations. Assuming a Bjorken-type longitudinal expansion and including the influence of temperature dependent masses on the expansion, we determine the total number of $c \bar{c}$ pairs produced in the quarkgluon plasma phase. We calculate the charm production rate at leading order with massive gluons and quarks and compare our result to charm production by massless partons. We consider two different scenarios for the initial conditions, a parton gas with a rather long kinetic equilibration time and a minijet gas with a short equilibration time. In a parton gas, assuming $m_{c}=1.2 \mathrm{GeV}$, we obtain a substantial enhancement over the thermal charm rate from massless quarks and gluons, up to 4.9 secondary charm quark pairs in $\mathrm{Au}+\mathrm{Au}$ collisions at $\mathrm{RHIC}$ and 245 charm pairs in $\mathrm{Pb}+\mathrm{Pb}$ collisions at the LHC.
\end{abstract}

LBNL-39984 Submitted to Phys. Rev. C.

\footnotetext{
${ }^{0 \star}$ This work was supported in part by the Director, Office of Energy Research, Division of Nuclear Physics of the Office of High Energy and Nuclear Physics of the U. S. Department of Energy under Contract Number DE-AC03-76SF0098.
} 


\section{INTRODUCTION}

Two decades ago, studies of quantum chromodynamics (QCD) predicted that quarks and gluons can be liberated from hadrons and, at appropriately high energy densities, a new state of the matter, the quark-gluon plasma (QGP) will be formed [1]. LatticeQCD calculations have shown that the critical energy density of this phase transition is $\varepsilon_{c} \approx 2-3 \mathrm{GeV} / \mathrm{fm}^{3}$. The critical temperature is $T_{c} \approx 260 \mathrm{MeV}$ for a pure gluon plasma while $T_{c} \approx 150 \mathrm{MeV}$ when dynamical light quarks are included [2]. These conditions are expected to be produced in ultrarelativistic nucleus-nucleus collisions. Recent experiments have not unambiguously proved QGP formation at the CERN SPS where $\sqrt{s}=17-20$ A $\mathrm{GeV}$, but in heavy-ion collisions at RHIC $(\sqrt{s}=200 \mathrm{~A} \mathrm{GeV})$ and $\mathrm{LHC}(\sqrt{s}=5.5 \mathrm{~A} \mathrm{TeV})$ the required energy densities should be produced.

At ultrarelativistic energies, nucleus-nucleus collisions can be described by parton interactions in perturbative QCD inspired models [3, 4]. In this framework, hard and semi-hard scatterings among partons dominate the reaction dynamics. These collisions are able to drive the parton system toward thermal equilibrium very effectively [5, 6, 7], but chemical equilibrium may not be established [6, 7]. Equilibration strongly depends on the actual parton densities in the colliding nuclei and the microscopic processes on the parton level. The behavior of the parton densities at small $x$ is not fully established: newer parametrizations of the parton densities already greatly increase the energy density of the initial state [7] and the introduction of softer semi-hard scatterings (a smaller $p_{T}$ cutoff on the minijet cross section) could yield a parton system very close to equilibrium a short time after the primary collisions.

During the time-evolution of the parton system, in parallel with the thermalization, many collective phenomena can appear. The massless color degrees of freedom, the quarks and gluons, are strongly interacting, as described in the framework of finite temperature QCD [8]. In this theory, temperature dependent thermal quark and gluon masses are introduced which can dramatically affect the phenomenological description of the time evolution. Massive fermionic and bosonic excitations can be derived in many ways, e.g. by considering leading order medium effects on the QCD self-energy [9]. Since the behavior of the QGP and its high-temperature excitations are not well understood, it is challenging to find measurable tests of this behavior in heavy ion collisions.

In general, open charm [10, 11, 12, 13, 14, direct photons and dilepton production 15] can be used as direct probes of the early parton dynamics and the evolution of the QGP. Primary charm production from the initial nucleon-nucleon collisions has been calculated and can be substantial [10], especially when up-to-date parameterizations of the parton densities which increase at low $x$, are used [11, 13. In the most recent calculation of initial charm production [13] in $\mathrm{Au}+\mathrm{Au}$ collisions, extrapolated from $p p$ collisions, a total of 9 (450) initial $c \bar{c}$ pairs were produced at RHIC (LHC), primarily due to the behavior of the parton densities at low $x$. As shown in ref. [13], shadowing can reduce the

yield in the central region by up to a factor of two, depending on the $c \bar{c}$ pair mass and transverse momentum. 
The amount of secondary charm production in the QGP phase is an open question [12, 13, 14, 16. If the parton system is dilute [6, 14], secondary charm production would be suppressed because of the low parton fugacities. Furthermore, at RHIC the charm mass is 2-3 times larger than the expected temperature scale, $T \sim 0.35-0.55 \mathrm{GeV}$, and much greater than the bare light quark masses. Thus in this case, even in a fully equilibrated plasma charm production is not significantly enhanced. At the higher LHC energy, the predicted initial temperatures, $T \approx 0.7-1 \mathrm{GeV}$, are close to the charm mass. One can then expect a larger thermal charm production rate than at lower energies, perhaps comparable to the initial yield.

Charm production in the plasma by massless quarks and gluons is small because the massless partons must be very energetic to overcome the $c \bar{c}$ pair mass threshold. However, the effective thermal quark and gluon masses generated by the plasma could significantly enhance the thermal charm yield, perhaps even approaching the initial yield after shadowing has been included. This additional charm production is a possible probe of massive excitations in the QGP which could test the level of thermalization and the evolution of the quark gluon plasma. Furthermore a large charm quark multiplicity would favor the production of exotic phenomena such as multi-charm hadron production [17].

The influence of massive gluons on strangeness production has already been investigated [18] and found to have a negligible influence on the total strangeness production rate. However, that calculation considered massless light quarks and a temperature region, $T \approx 200-250 \mathrm{MeV}$, only slightly above $T_{c}$. Here we perform a similar calculation but with massive quarks as well as massive gluons and with the higher initial temperatures obtained from more recent estimates of the initial conditions [7, 19]. Under these circumstances, the thermal quark and gluon masses can generate a significant increase in secondary charm production.

In this paper we investigate the thermal charm production rate in a fully equilibrated QGP with massive quark and gluon degrees of freedom. We compare our results with those from zero mass quarks and gluons. We will assume both a pure gluon gas and a quark-gluon plasma. We also distinguish between parton gas and minijet gas initial conditions. We obtain the total number of $c \bar{c}$ pairs produced during the lifetime of the plasma by assuming a Bjorken-type longitudinal expansion for $\mathrm{Au}+\mathrm{Au}$ collisions at $\mathrm{RHIC}$ and $\mathrm{Pb}+\mathrm{Pb}$ collisions at $\mathrm{LHC}$. The influence of temperature dependent masses on the expansion and the speed of sound is also considered in detail.

\section{EFFECTIVE QUARK AND GLUON MASSES}

In a strongly-interacting QGP, collective fermionic and bosonic excitations appear, as also seen in QED. These quasi-particles can be interpreted as massive quarks and massive gluons. The propagators are modified in the medium because the color charges are dressed by the interaction with their neighbors in the plasma phase. The effective masses are related to the properties of the QGP and can thus be characterized by their temperature 
and density dependence. Using the high temperature approximation [20] or, equivalently, the hard thermal loop approximation [21] which ensures a gauge invariant treatment, one can obtain the following effective equilibrium masses at zero chemical potential, $\mu=0$, for quarks and gluons massless at $T=0$ [8]:

$$
\begin{aligned}
& m_{q, \mathrm{th}}^{2}(T)=\frac{g^{2} T^{2}}{6} \\
& m_{g, \mathrm{th}}^{2}(T)=\frac{g^{2} T^{2}}{3}\left(1+\frac{N_{f}}{6}\right) .
\end{aligned}
$$

We assume that the equilibrated QGP contains light $u, d$, and $s$ quarks and anti-quarks so that $N_{f}=3$ and $m_{g, \text { th }}=g T / \sqrt{2}$. Since the strange quark has a non-zero bare mass, we consider an approximate effective mass, $\hat{m}_{s}$, where

$$
\hat{m}_{s}=\sqrt{m_{s, 0}^{2}+m_{q, \mathrm{th}}^{2}},
$$

and $m_{s, 0}=150 \mathrm{MeV}$. We neglect the small current mass of the light quarks so that $\hat{m}_{q} \approx m_{q, \mathrm{th}}$. The effective mass of the gluon is similar, $\hat{m}_{g}=m_{g, \text { th}}$. Charm quarks are assumed to be produced with their bare mass and dressed later. The hadronization of charm quarks does not influence the charm production rate.

The temperature-dependent running coupling constant is

$$
g^{2}\left(\frac{T}{T_{c}}\right)=\frac{24 \pi^{2}}{\left(33-2 N_{f}\right) \ln \left[\left(19 T_{c} / \Lambda_{\overline{\mathrm{MS}}}\right)\left(T / T_{c}\right)\right]} .
$$

where in $\mathrm{SU}(3)$ gauge theory, $T_{c} / \Lambda_{\overline{\mathrm{MS}}}=1.78 \pm 0.03$ [22]. With this dependence, the thermal gluon and quark masses increase approximately linearly with in the temperature, $m_{g, \text { th }} \approx\left(130 T / T_{c}+36\right) \mathrm{MeV}$ and $m_{q, \text { th }} \approx m_{g, \text { th }} / \sqrt{3}$, where $T_{c}=150 \mathrm{MeV}$. Then at $T \approx 500 \mathrm{MeV}, \hat{m}_{g} \approx 470 \mathrm{MeV}$ while at the lower temperature assumed earlier [18], $T \approx 300 \mathrm{MeV}, \hat{m}_{g} \approx 300 \mathrm{MeV}$. In ref. [18, 23] massive gluons were introduced by reproducing lattice-QCD energy density and pressure results [24]. The effective gluon mass, assumed constant, was $M_{g} \approx 450-500 \mathrm{MeV}$ for $1.2<T / T_{c}<2.4,30-40 \%$ larger than our value in the same region.

\section{CHARM QUARK PRODUCTION}

The processes relevant for $c \bar{c}$ pair production in the quark-gluon plasma are the following:

$$
\begin{aligned}
& q+\bar{q} \longrightarrow c+\bar{c} \\
& s+\bar{s} \longrightarrow c+\bar{c} \\
& g+g \longrightarrow c+\bar{c}
\end{aligned}
$$

An analogy can be drawn between charm and strangeness production by the plasma. In

early calculations of strangeness production, processes (5) and (7) were treated at leading 
order with bare quark and gluon masses [25]. On the other hand, effective quark masses were used as infrared cut-offs simultaneously removing electric and magnetic infrared singularities, see e.g. ref. [5]. Effective gluon masses were included in calculations of chemical equilibration [6] and strangeness production [18] in the QGP, and in calculations of radiative energy loss in a parton gas [26]. In these cases the bare Green's functions were used with the effective gluon mass but the quarks were not dressed. Here we combine these approximations, using the bare Green's functions containing dressed quarks and gluons and calculating the production rates in perturbative QCD at leading order.

In the calculation of strangeness production with massive gluons [18 one additional process was included, the decay of massive gluons. From the thermal gluon mass, eq. (2), and the temperature dependent running coupling constant, eq. (4), we estimate that the threshold for $g \rightarrow c \bar{c}, \hat{m}_{g}>2 m_{c}$, will be reached only at $T \approx 20 T_{c}$. We therefore neglect this channel. We can also assume that the annihilation of produced $c \bar{c}$ pairs is negligible. The total charm production rate is thus:

$$
\frac{d N_{c \bar{c}}}{d \tau}=\left[A_{g}(T)+2 A_{q}(T)+A_{s}(T)\right] \cdot V(\tau)
$$

where

$$
A_{i}(T)=\frac{1}{n_{i} !} \int d s \int 2 \sigma_{i}(s) \sqrt{s\left(s-4 \hat{m}_{i}^{2}\right)} \frac{f_{i}\left(E_{1}\right)}{2 E_{1}} \frac{f_{\bar{i}}\left(E_{2}\right)}{2 E_{2}} \frac{d^{3} p_{1}}{(2 \pi)^{3}} \frac{d^{3} p_{2}}{(2 \pi)^{3}}
$$

and $n_{i}$ is the number of identical particles in the initial state. We calculate $A_{q}$ and $A_{s}$ separately since $\hat{m}_{q} \neq \hat{m}_{s}$. The Bose distribution is used for gluons, $f_{g}(E)=\left(e^{E / T}-1\right)^{-1}$, and the Fermi distribution is used for quarks, $f_{q, \bar{q}}(E)=\left(e^{E / T}+1\right)^{-1}$. We consider a symmetric QGP with zero baryon and strangeness chemical potential, $\mu_{q}=\mu_{s}=0$. The total production cross section for each channel, $\sigma_{i}$, is calculated at center-of-mass energy $\sqrt{s}$.

The gluon-gluon fusion and quark-antiquark annihilation rates can be written in the Lorentz-invariant form

$$
\begin{aligned}
A_{i}(T)= & \frac{1}{n_{i} !} \int d s \int \frac{d^{4} p_{1}}{(2 \pi)^{3}} \int \frac{d^{4} p_{2}}{(2 \pi)^{3}} 2 \sigma(s) \sqrt{s\left(s-4 m_{i}^{2}\right)} \delta\left(s-\left(p_{1}+p_{2}\right)^{2}\right) \\
& \cdot \delta\left(p_{1}^{2}-m_{i}^{2}\right) \delta\left(p_{2}^{2}-m_{i}^{2}\right) f_{i}\left(E_{1}\right) f_{i}\left(E_{2}\right)
\end{aligned}
$$

with four momenta $p_{1}=\left(E_{1}, \overrightarrow{\mathbf{p}_{\mathbf{1}}}\right)$ and $p_{2}=\left(E_{2}, \overrightarrow{\mathbf{p}_{\mathbf{2}}}\right)$. Following ref. 18 this integral can be rewritten as

$$
A_{i}(T)=\frac{1}{32 \pi^{4}} \frac{1}{n_{i} !} \int_{4 m_{i}^{2}}^{\infty} d s \sigma_{i}(s) s\left(s-4 m_{i}^{2}\right) \sum_{l=2}^{\infty} \sum_{k=-l+2}^{l-2}( \pm 1)^{l} \frac{K_{1}\left(a_{k l}\right)}{a_{k l}}
$$

where the sum over index $k$ is incremented by 2 , with the + sign used for bosons and the - sign for fermions. The modified imaginary Bessel function, $K_{1}\left(a_{k l}\right)$, has the argument

$$
a_{k l}=\frac{\sqrt{s}}{2 T} \sqrt{l^{2}-\left(1-\frac{4 m_{i}^{2}}{s}\right) k^{2}}
$$


The use of a simple Boltzmann distribution instead of the Bose and Fermi distributions is equivalent to reducing the sum to only the $l=2, k=0$ term with $a_{02}=\sqrt{s} / T$. At high temperatures and high effective quark and gluon masses, the Boltzmann distribution is a good approximation. However, here we will use the full sum.

Once the time evolution of the volume, $V(\tau)$, and the temperature, $T(\tau)$, are determined, we can calculate the appropriate quark and gluon masses and the total charm pair production rate.

\subsection{ELEMENTARY CROSS SECTIONS}

Here we explain the calculation of the total $c \bar{c}$ subprocess production cross sections for massive quarks and gluons. As discussed earlier, we neglect $c \bar{c}$ pair annihilation as well as the decay of massive gluons.

When the light quarks have an effective mass, the square of the matrix element for processes (4) and (5) is

$$
\left|M_{i}\right|^{2}=2 g^{4} \frac{\operatorname{Tr}\left[\gamma^{\mu}\left(\not p-\hat{m}_{i}\right) \gamma^{\nu}\left(\not q+\hat{m}_{i}\right)\right] \operatorname{Tr}\left[\gamma_{\mu}\left(\not p_{1}-m_{c}\right) \gamma_{\nu}\left(\not p_{2}+m_{c}\right)\right]}{\left(s-\hat{m}_{g}^{2}+\Gamma^{2} / 4\right)^{2}+\Gamma^{2} \hat{m}_{g}^{2}},
$$

where $p^{\mu}$ and $q^{\mu}$ are the four-momenta of the incoming quarks, $i=u, d$ and $s$, and $p_{1}^{\mu}$ and $p_{2}^{\mu}$ are the four-momenta of the outgoing $c$ and $\bar{c}$ quarks. The propagator has been modified by the finite mass and width of the gluons as in ref. [18. After evaluation of the traces, the square of the matrix element can be expressed as

$$
\left|M_{i}\right|^{2}=d_{i}^{2} \pi^{2} \alpha_{S}^{2} \frac{64}{9} \frac{\left(m_{c}^{2}+\hat{m}_{i}^{2}-t\right)^{2}+\left(m_{c}^{2}+\hat{m}_{i}^{2}-u\right)^{2}+\left(2 m_{c}^{2}+2 \hat{m}_{i}^{2}\right) s}{\left(s-\hat{m}_{g}^{2}+\Gamma^{2} / 4\right)^{2}+\Gamma^{2} \hat{m}_{g}^{2}}
$$

where $g^{2}=4 \pi \alpha_{S}$ and $d_{i}=6$ for the spin and color degrees of freedom for each flavor. Note that eq. (13) does not include the usual average over the initial spin and color while eq. (14) does include these factors.

The total cross section is obtained from the $t$ integration of $\left|M_{i}\right|^{2} \mid$,

$$
\sigma_{i}(s)=\frac{1}{16 \pi s\left(s-4 \hat{m}_{i}^{2}\right)} \int_{t_{-}}^{t_{+}} d t\left|M_{i}\right|^{2}
$$

with the limits

$$
t_{ \pm}=-\left(\frac{\sqrt{s-4 \hat{m}_{i}^{2}}}{2} \mp \frac{\sqrt{s-4 m_{c}^{2}}}{2}\right)^{2}
$$

where the sum of the Mandelstam invariants $s, t$ and $u$ is

$$
s+t+u=2 \hat{m}_{i}^{2}+2 m_{c}^{2} .
$$


For gluon fusion, (7), the total cross section can be obtained from the integral

$$
\sigma_{g}(s)=\frac{1}{16 \pi s\left(s-4 \hat{m}_{g}^{2}\right)} \int_{t_{-}}^{t_{+}} d t\left|M_{s}+M_{u}+M_{t}\right|^{2}
$$

with the integration limits

$$
t_{ \pm}=-\left(\frac{\sqrt{s-4 \hat{m}_{g}^{2}}}{2} \mp \frac{\sqrt{s-4 m_{c}^{2}}}{2}\right)^{2} .
$$

Detailed calculations of the invariant matrix elements, $M_{s}, M_{u}$ and $M_{t}$, are given in ref. [18] for $s \bar{s}$ production by transverse gluons of constant mass. The same expressions can be used here with the change $m_{s} \rightarrow m_{c}$. The sum of the Mandelstam invariants is now

$$
s+t+u=2 \hat{m}_{g}^{2}+2 m_{c}^{2} .
$$

We use two different values for the charm quark mass: $m_{c}=1.2 \mathrm{GeV}$ and $m_{c}=$ $1.5 \mathrm{GeV}$. The lower value was found to produce agreement between charm production calculated to NLO [11] and $p p$ total cross section data. This value has also been used in recent estimates of the charm contribution to the dilepton yield [13]. The larger value is somewhat more standard [12, 10, 14] and, at energies near the charm production threshold, allows an all-order resummation of soft and virtual gluon corrections [27]. For smaller charm quark masses, the series cannot be resummed.

\subsection{THE HYDRODYNAMICAL MODEL}

We now discuss our calculations of the equation of state and the time evolution of the plasma. The pressure of an ideal gas of massive particles is

$$
P=\sum_{i} \frac{g_{i}}{6 \pi^{2}} \int_{0}^{\infty} \frac{d k k^{4}}{\sqrt{k^{2}+\hat{m}_{i}^{2}}}\left[\exp \left(\beta \sqrt{k^{2}+\hat{m}_{i}^{2}}\right) \mp 1\right]^{-1},
$$

where $i=g, u, \bar{u}, d, \bar{d}, s, \bar{s}$. In the case of a symmetric plasma, $\mu_{u}=\mu_{d}=\mu_{s}=0$, and we have $P_{i}=P_{\bar{i}}$. Since $\hat{m}_{i}$ is temperature dependent, the energy density, $\epsilon=T d P / d T-P$, has an additional term proportional to the mass gradient

$$
\epsilon=\sum_{i} \frac{g_{i}}{2 \pi^{2}} \int_{0}^{\infty} \frac{d k k^{2}}{\sqrt{k^{2}+\hat{m}_{i}^{2}}}\left[\exp \left(\beta \sqrt{k^{2}+\hat{m}_{i}^{2}}\right) \mp 1\right]^{-1}\left(k^{2}+\hat{m}_{i}^{2}-\hat{m}_{i} T \frac{d \hat{m}_{i}}{d T}\right) .
$$

We assume a simple longitudinal expansion and follow the time evolution through the entropy

$$
s(\tau)=s\left(\tau_{0}\right)\left(\tau_{0} / \tau\right) .
$$

We also calculate the square of the sound speed, defined as

$$
c_{s}^{2}=\frac{d P / d T}{d \epsilon / d T},
$$


where $d P / d T=s=(\epsilon+P) / T$ as a check on how closely the evolution follows that of an ideal massless gas with $c_{s}^{2}=1 / 3$. The temperature gradient of the energy density is

$$
\begin{aligned}
\frac{d \epsilon}{d T}= & \sum_{i} \frac{1}{T} \frac{g_{i}}{2 \pi^{2}} \int_{0}^{\infty} \frac{d k}{\sqrt{k^{2}+\hat{m}_{i}^{2}}}\left[\exp \left(\beta \sqrt{k^{2}+\hat{m}_{i}^{2}}\right) \mp 1\right]^{-1}\left\{\left(k^{2}+\hat{m}_{i}^{2}\right)^{2}\right. \\
& +\left(k^{2}+\hat{m}_{i}^{2}\right)\left(3 k^{2}-2 \hat{m}_{i} T \frac{d \hat{m}_{i}}{d T}\right) \\
& \left.+T^{2}\left[\left(\hat{m}_{i} \frac{d \hat{m}_{i}}{d T}\right)^{2}-k^{2}\left(\left(\frac{d \hat{m}_{i}}{d T}\right)^{2}+\hat{m}_{i} \frac{d^{2} \hat{m}_{i}}{d T^{2}}+2 \frac{\hat{m}_{i}}{T} \frac{d \hat{m}_{i}}{d T}\right)\right]\right\} .
\end{aligned}
$$

Note that the above expressions reduce to $c_{s}^{2}=1 / 3$ when $\hat{m}_{i}$ is constant.

The mass gradients can be expressed rather simply when $\hat{m}_{i}=c g(T) T$ and the current quarks and gluons are massless. In this case we have

$$
\begin{aligned}
\frac{d \hat{m}_{i}}{d T} & =\frac{\hat{m}_{i}}{T}\left[1-\frac{1}{2}\left(\frac{\hat{m}_{i}}{c T}\right)^{2}\right] \\
\frac{d^{2} \hat{m}_{i}}{d T^{2}} & =-\frac{1}{2} \frac{\hat{m}_{i}^{3}}{c^{2} T^{4}}\left[1-\frac{3}{2}\left(\frac{\hat{m}_{i}}{c T}\right)^{2}\right] .
\end{aligned}
$$

For the strange quark, $\hat{m}_{s}=\sqrt{m_{s, 0}^{2}+m_{q}^{2}(T)}$, the derivatives are

$$
\begin{aligned}
\frac{d \hat{m}_{s}}{d T} & =\frac{1}{\hat{m}_{s}} \frac{d m_{q}}{d T} \\
\frac{d^{2} \hat{m}_{s}}{d T^{2}} & =-\frac{1}{\hat{m}_{s}^{3}}\left(\frac{d m_{q}}{d T}\right)^{2}+\frac{1}{\hat{m}_{s}} \frac{d^{2} m_{q}}{d T^{2}}
\end{aligned}
$$

where $d m_{q} / d T=d \hat{m}_{i} / d T$ as above. Including effective quark and gluon masses tends to slow the evolution of the system as well as increase the $c \bar{c}$ rate.

We need to fix the space-time volume to calculate the absolute number of produced charm pairs. The particle number is obtained from

$$
N=\int n^{\mu} d \sigma_{\mu}=\rho \pi R^{2} \tau \int_{-\eta^{*}}^{\eta^{*}} d \eta \cosh \eta=\rho V
$$

where $V=2 \pi R^{2} \tau \sinh \eta^{*}$. To determine the maximum space-time extent of the plasma in rapidity as a function of $\tau$, we use the total available energy as a rough estimate:

$$
\begin{aligned}
E_{\mathrm{tot}} & =2 A E_{\mathrm{beam}}=\int T^{0 \mu} d \sigma_{\mu} \\
& =\pi R^{2} \tau \int_{-\eta^{*}}^{\eta^{*}} d \eta\left[\epsilon \cosh ^{2} \eta+P \sinh ^{2} \eta\right] .
\end{aligned}
$$

From a comparison of the calculated $E_{\text {tot }}$ with the available energy, $2 A E_{\text {beam }}$, we can determine the value of $\eta^{*}$ and calculate the volume. With this volume, the charm yield is calculated from eq. (8). 


\section{TIME EVOLUTION OF THE SYSTEM}

We will consider the hydrodynamical evolution of a fully equilibrated plasma with two different sets of initial conditions, $T_{0}$ and $\tau_{0}$, for RHIC and LHC collisions.

The first parameter set is based on the parton gas model derived from the HIJING Monte Carlo code [3]. The kinetic equilibration time, relatively long, is reached when the momentum distributions are locally isotropic due to elastic scatterings and the expansion of the system, $\tau_{0} \sim 0.5-0.7 \mathrm{fm}$. We use $T_{0}=550 \mathrm{MeV}$ and $\tau_{0}=0.7 \mathrm{fm}$ at RHIC and $T_{0}=820 \mathrm{MeV}, \tau_{0}=0.5 \mathrm{fm}$ at LHC, as in the ideal case described in ref. 119. With this model we do not distinguish between a gluon gas and a quark-gluon plasma in the estimate of $T_{0}$.

The second set of initial conditions was determined from estimates of minijet production [7]. In this case, the momentum scale of the minijet calculation sets the initial time, $\tau_{0} \sim 1 / p_{T} \leq 1 / p_{0}$, and the minijet yield determines the initial temperature. For a typical value of the momentum scale, $p_{0} \sim 2 \mathrm{GeV}, \tau_{0} \sim 0.1 \mathrm{fm}$. Because the minijet yield depends on the composition of the system, the initial temperature depends on the partonic degrees of freedom. Since minijets predominantly produce gluons, if we consider only a gluon gas, the highest $T_{0}$ is obtained, $T_{0}=445 \mathrm{MeV}$ at RHIC and $1140 \mathrm{MeV}$ at LHC. When light quark production is included, the number density increases but $T_{0}$ decreases to $360 \mathrm{MeV}$ at RHIC and $900 \mathrm{MeV}$ at LHC. The plasma resulting from the early equilibration time and high temperature is referred to hereafter as a minijet gas.

We compare and contrast the evolution of the plasma with the parton gas and the minijet gas in $\mathrm{Au}+\mathrm{Au}$ collisions at $\mathrm{RHIC}$ in fig. 1 and in $\mathrm{Pb}+\mathrm{Pb}$ collisions at the $\mathrm{LHC}$ in fig. 2. In each case, we show results for a pure gluon system and a quark-gluon system both with massless and massive partons. The time evolution of the temperature, energy density, square of the sound speed in the medium and the volume of the plasma are given in each case.

The parton gas has a longer lifetime due to the longer equilibration time. The temperature evolution is shown in figs. 1(a) and 2(a) for the parton gas and in 1(b) and 2 (b) for the minijets. The difference between the temperature evolution with gluons alone and for the quark-gluon gas is seen to be small, independent of whether or not the partons are massive. The evolution slows when the parton masses are finite. We have cut off the evolution at $T_{c}=150 \mathrm{MeV}$. The parton gas, with its slower evolution, remains above $T_{c}$ for $\tau \leq 10 \mathrm{fm}$. At RHIC energies the temperature of the minijet gas drops below $T_{c}$ at $\tau \approx 1.5 \mathrm{fm}$ in the quark-gluon system. The pure gluon system, with its larger $T_{0}$, remains above $T_{c}$ for at least $2.5 \mathrm{fm}$ at RHIC. For both the parton and minijet gas, the higher initial temperatures at the LHC make the finite parton masses more effective in slowing the evolution, in part because the effective parton masses are larger for the higher temperature.

The increase in the number of degrees of freedom between a pure gluon gas and a quark-gluon gas is clearly reflected in the difference between the energy densities of the 
two systems, shown in figs. $1(\mathrm{c}-\mathrm{d})$ and $2(\mathrm{c}-\mathrm{d})$. Note that in both cases at RHIC, the energy density of the massive gluon gas is actually reduced relative to the massless gluon gas. This is due to the temperature gradient of the gluon mass. A similar effect is also observable in the minijet quark-gluon gas. In all these cases, the temperature is not large compared to the effective masses, causing the reduction. At the much higher temperatures of the LHC, although the effective masses are increased, the energy density is increased by the inclusion of the finite parton masses. We note that at RHIC, the energy density of the minijet gas actually drops below $1 \mathrm{GeV} / \mathrm{fm}^{3}$ for $\tau \geq 1 \mathrm{fm}$, even though the temperature remains above $T_{c}$ until $\tau \approx 1.5 \mathrm{fm}$ in the quark-gluon system and until $\tau \approx 2.5 \mathrm{fm}$ in the gluon gas. This suggests that the assumption of an equilibrated minijet gas at RHIC is perhaps questionable.

The speed of sound in the medium remains close to that of an ideal gas, as shown in figs. 1(e-f) and 2(e-f). As expected, in the massless gluon case, $c_{s}^{2} \equiv 1 / 3$. The deviation of the dot-dashed curve from the ideal gas result is due to the finite current strange quark mass even though the light quarks and gluons are massless. In this case, the system moves further from the ideal gas behavior at later times as the temperature becomes comparable to $m_{s}$. When the system is composed of massive gluons only, the deviation from an ideal gas is largest because the effective gluon mass is larger than the effective quark masses. The addition of the lighter massive quarks into the system tends to bring the sound speed closer to the ideal gas value. At the LHC, the higher temperature keeps the system closer to the ideal gas limit than at RHIC.

The volume of the system, crucial to the determination of the charm yield, increases as shown in figs. $1(\mathrm{~g}-\mathrm{h})$ and $2(\mathrm{~g}-\mathrm{h})$. Since the volume depends on the rapidity extent of the plasma, the relatively lower energy density and pressure of the gluon gas require a larger spatial extent to ensure that the energy of the system is equal to $E_{\text {tot }}$. Because we have changed the initial conditions according to the composition of the minijet gas, the volume changes less than the parton gas volume.

\section{TOTAL CHARM YIELD}

In this section, we present our results on the $c \bar{c}$ production rates. The parton gas produces the largest $c \bar{c}$ yield because of the slower time evolution demonstrated in the previous section. Since the time evolution of the quark and gluon effective masses determines the relative enhancement of charm production by the massive excitations, we show the time dependence of the effective masses in a quark-gluon system in fig. 3. The finite current strange quark mass at zero temperature results in a slightly higher effective strange quark mass compared to the effective light quark mass. It also reduces the time dependence of the strange quark effective mass. The larger slope of the gluon effective mass as a function of temperature results in a faster decrease in gluon mass as a function of time. We have shown the effective masses for as long as $T>T_{c}$ - the minijet gas at RHIC is at $T_{c}$ when $\tau \sim 1.5 \mathrm{fm}$. At later times, the finite masses become less effective for producing charm. 
We remark that the running coupling constant, $g$, is also a function of time. The weaker coupling at later times also reduces the yield.

The initial rate is approximately a factor of two larger when the quark degrees of freedom are included. At the beginning of the evolution, the rate is nearly independent of the initial parton mass. However, since the system cools more slowly with massive initial partons, the rate is larger at later times in the massive case. The number of $c \bar{c}$ pairs produced during the lifetime of the plasma is found by multiplying the rate, eq. (11), by the volume from eq. (30). In most cases, charm production only occurs during the early part of the evolution. At RHIC, production by the parton gas is essentially over after $\sim 3 \mathrm{fm}$ while production from the minijet gas is ended by $\sim 0.5 \mathrm{fm}$. Note however, that $c \bar{c}$ pairs continue to be produced at much later times when the partons are massive, especially at the LHC. This is particularly true for the massive quark-gluon gas.

The charm production rates and the number of produced $c \bar{c}$ pairs as a function of time at RHIC are given in fig. 4 for $m_{c}=1.2 \mathrm{GeV}$ and fig. 5 for $m_{c}=1.5 \mathrm{GeV}$. The final charm pair yield in both cases is given in Table 1. The parton gas results are given in fig. 4(a-b) and 5(a-b). Here, although the production rate is larger when both quarks and gluons are included, the final number of $c \bar{c}$ pairs produced during the evolution of the system does not strongly depend on the composition of the plasma at RHIC because the larger rate is compensated by a correspondingly smaller volume. The yield from a massless quark-gluon gas is about 15\% larger than that from a massless gluon gas. For massive initial quarks and gluons, the difference in the composition changes the yield by only $8 \%$, as seen in Table 1 . When $m_{c}=1.2 \mathrm{GeV}$, the yield is increased $44 \%$ for a massive gluon gas relative to a massless gluon gas and $32 \%$ in the massive quark-gluon system. If $m_{c}=1.5 \mathrm{GeV}$, the enhancement due to the massive partons is reduced to $36 \%$ for the gluons alone and to $29 \%$ for the quark-gluon gas. The yield at the lower mass is about 3.5 times larger than when $m_{c}=1.5 \mathrm{GeV}$. Some of the enhancement can be accounted for by the slower temperature evolution. After the system has evolved for $10 \mathrm{fm}$, the temperature of the massive gluon gas is approximately $10 \%$ larger than the massless gas. The difference is $8 \%$ when the quarks are included. Note particularly that the 5 thermal $c \bar{c}$ pairs produced with $m_{c}=1.2 \mathrm{GeV}$ is only a factor of two less than that expected from the initial production at this energy 13 .

The yield is much smaller from a minijet gas due to the shorter equilibration time and the lower initial temperature. (In the minijet gas, $T_{0}$ is reduced $24 \%$ for gluons alone and $53 \%$ for a quark-gluon system relative to the initial temperature of the parton gas.) In the minijet gas, the charm yield is reduced by a factor of 60-70 (for gluons) and 250-300 (for quarks and gluons) compared to the parton gas. The thermal charm yield from the minijet gas is thus negligible compared to the initial charm rate [13]. The influence of the massive partons is also reduced for the minijet gas. For $m_{c}=1.2 \mathrm{GeV}$, the yield is only increased over the massless case by $23 \%$ in a gluon gas and $14 \%$ in a quark-gluon gas. The influence of the charm mass on the yield is also stronger for the minijet gas - the yield decreases by a factor of 4-5 with the larger charm mass. 
The enhancement due to the effective parton mass is more substantial at the LHC, as seen in figs. 6 and 7 and in Table 2. The massive quarks and gluons have a more significant effect on the temperature evolution of the parton gas. The temperature is $27 \%$ higher after $10 \mathrm{fm}$ with massive gluons and $20 \%$ higher for massive quarks and gluons. The enhancement of the yield is also larger: when $m_{c}=1.2 \mathrm{GeV}$, the yield is $94 \%$ larger in the gluon gas and $70 \%$ higher in the quark-gluon gas. Increasing the charm mass only reduces the total yield by a factor of 2.5, thus the yield is less dependent on the charm mass at the higher temperature. Note that here the charm yield from the massive quark-gluon system, 250 pairs after $10 \mathrm{fm}$, is similar to the initial nucleon-nucleon rate 13..

The minijet gas is more effective at producing charm at LHC than at RHIC. Although the initial time remains short, $T_{0}$ is $40 \%$ larger in the minijet gluon gas and $10 \%$ larger in the minijet quark-gluon gas than in the parton gas. Therefore the minijet charm yield is only a factor of two to six smaller than the parton gas yield and the minijet thermal charm yield also becomes a significant fraction of the initial production. Note also that the enhancement due to the massive partons is largest in the minijet gluon gas-a factor of 2.5 increase over that from a gas of massless gluons because the temperature of the massive gluon gas is $50 \%$ larger.

In Ref. [13], with an ideal, massless, quark-gluon plasma, one thermal charm pair was found at RHIC and 26 charm pairs at LHC with $m_{c}=1.2 \mathrm{GeV}$. In that work, the initial temperature was nearly the same as the parton gas $T_{0}$ used here but the initial time was closer to that of the minijet gas, $\tau_{0} \sim\left(3 T_{0}\right)^{-1}$. However, a larger, constant, $g^{2}$ kept the yield from being reduced. When initial conditions identical to those used here are chosen for the calculation of Ref. [13], the results are quite similar. While the details of the expansion are somewhat different, we have checked that our massless charm production cross sections are in exact agreement with those in Ref. [13.

To study the dependence of the enhancement on the charm pair mass, we also calculate the thermal charm pair mass distributions for RHIC in fig. 8 and for the LHC in fig. 9. Generally, the thermal charm mass distributions are steeper than those charm pairs produced in the initial nucleon-nucleon interactions. (See ref. [13] for the initial charm pair mass distributions.) The shapes of the mass distributions for each of the four cases we have studied with our two sets of initial conditions are quite similar, especially for the parton gas where $T_{0}$ is the same in all cases. The shapes of the distributions from the minijet gas at RHIC are also similar although the initial yield is larger from the gluon gas because of its higher $T_{0}$. The enhancement is generally largest for low mass charm pairs, not far above threshold. At LHC, where the enhancement is greatest, the distributions with the massive excitations included approach the mass distributions for the massless case only at $M \sim 8-10 \mathrm{GeV}$. 


\section{DISCUSSION}

We have investigated a new mechanism for enhancing thermal charm production by a quark-gluon plasma: massive excitations in the plasma state. We chose two different sets of initial conditions, a parton gas and a minijet gas, and calculated the thermal charm yield from each for both massless and massive quarks and gluons. In our calculation we assumed that the system stayed in thermal and chemical equilibrium during the expansion. Therefore, our charm yield is an upper bound on secondary charm production.

The largest charm production was found when a parton gas was assumed because the characteristic thermalization time was $\tau_{0}=0.5-0.7 \mathrm{fm}$. Then the parton gas lifetime was long, more then $10 \mathrm{fm}$. At RHIC we obtained a 30-40\% enhancement with massive gluons and quarks while at the LHC, a 50-100\% enhancement may be expected. With $m_{c}=1.2$ $\mathrm{GeV}$, the charm mass used in recent calculations, we obtained 4.9 secondary charm quark pairs at RHIC and 245 charm pairs at LHC. Note that these numbers, upper limits on secondary charm production, are similar to the expected initial charm production [13]. If we take $m_{c}=1.5 \mathrm{GeV}$, secondary charm pair production is reduced to 1.3 pairs at RHIC and 94 pairs at LHC.

The lifetime of the minijet gas was significantly shorter than the parton gas due to the short thermalization time, $\tau \sim 0.1 \mathrm{fm}$. This difference strongly reduced the charm yield from the minijet gas compared to the parton gas. The typical lifetime of the minijet gas was of order $2.5 \mathrm{fm}$ although at RHIC the temperature of the quark-gluon gas dropped below $T_{c}$ after only $1.5 \mathrm{fm}$. The fast expansion reduces the influence of the massive quasiparticles at RHIC where the initial temperature of the minijet gas was also smaller than the initial temperature of the parton gas. The enhancement was typically $20 \%$ with massive quarks and gluons but the total yield, 0.016 pairs from the quark-gluon gas, was very small compared to the initial charm rate. Although the expansion was also fast at the LHC, the much higher initial temperature, $T_{0} \approx 1 \mathrm{GeV}$, generated large charm production rates. With $m_{c}=1.2 \mathrm{GeV}$ we obtained 38 secondary charm quark pairs from a quark-gluon gas and 100 pairs from a gluon gas while with $m_{c}=1.5 \mathrm{GeV}$, the yield was 16 and 47 pairs respectively. Note that the minijet gluon gas result is only a factor of two smaller than the corresponding parton gas yield at LHC.

Our results show that, regardless of the initial conditions, the massive excitations of the quarks and gluons significantly enhance charm production by the plasma. Introducing thermal gluon and quark masses slowed the expansion of the system. The longer lifetime

of the plasma as well as the reduced threshold for charm production with massive quarks and gluons leads to an enhancement of charm production over the massless case. Thus charm enhancement in heavy ion collisions could be an excellent probe of the presence of collective excitations in the deconfined plasma. If such enhanced charm production, beyond that predicted from the initial nucleon-nucleon collisions is observed, it could be expected that other exotic phenomena due to massive quark and gluon excitations may be found. 


\section{Acknowledgments}

We would like to thank D. Rischke for enlightening discussions on the equation of state. The authors thank the Institute for Nuclear Theory at the University of Washington in Seattle for their hospitality. This work was supported in part by the Director, Office of Energy Research, Division of Nuclear Physics of the Office of High Energy and Nuclear Physics of the U. S. Department of Energy under Contract Number DE-AC03-76SF0098, by the National Scientific Research Fund (Hungary), OTKA No. T014213 and F019689

as well as by the U.S.-Hungarian Science and Technology Joint Fund, No. 378/93 and the Foundation for the Hungarian Science (MHB). 


\section{References}

[1] J.D. Bjorken, Phys. Rev. D27 (1983) 140; B. Müller, The Quark-Gluon Plasma, Springer, 1985.

[2] F. Karsch, Nucl. Phys. A590 (1995) 367c.

[3] X.N. Wang, M. Gyulassy, Phys. Rev. D44 (1991) 3501; Comput. Phys. Commun. 83 (1994) 307.

[4] K. Geiger, B. Müller, Nucl. Phys. B369 (1992) 600;

K. Geiger, Phys. Rev. D47 (1993) 133.

[5] E. Shuryak, Phys. Rev. Lett. 68 (1992) 3270;

L. Xiong, E. Shuryak, Phys. Rev. C49 (1994) 2207.

[6] T.S. Biró, E. van Doorn, B. Müller, M.H. Thoma, X.N. Wang, Phys. Rev. C48 (1993) 1275.

[7] K.J. Eskola, Nucl. Phys. A590 (1995) 383c;

K.J. Eskola, K. Kajantie and P.V. Ruuskanen, Phys. Lett. B332 (1994) 191.

[8] M. Le Bellac: Thermal Field Theory, Cambridge, 1996;

M. Thoma: Applications of High-Temperature Field Theory to Heavy Ion Collisions, hep-ph/9503400.

[9] U. Heinz, Phys. Rev. Lett. 51 (1983) 351; D. Vasak, M. Gyulassy, H.T. Elze, Ann. Phys. 173 (1987) 462; H.T. Elze, U. Heinz, Phys. Rep. 183 (1989) 81.

[10] B. Müller, X.N. Wang, Phys. Rev. Lett. 68 (1992) 2437.

[11] P.L. McGaughey et al., Int. J. Mod. Phys. A10 (1995) 2999.

[12] R. Vogt, B.V. Jacak, P.L. McGaughey and P.V. Ruuskanen, Phys. Rev. D49 (1994) 3345 .

[13] S. Gavin, P.L. McGaughey, P.V. Ruuskanen and R. Vogt, Phys. Rev. C54 (1996) 2606. (hep-ph/9604369)

[14] Z. Lin, M. Gyulassy, Phys. Rev. C51 (1995) 2177;

P. Lévai, B. Müller, X.N. Wang, Phys. Rev. C51 (1995) 3326.

[15] E. Shuryak, L. Xiong, Phys. Rev. Lett. 70 (1994) 2207.

M.T. Strickland, Phys. Lett. B331 (1994) 245.

[16] J. Rafelski, J. Letessier and A. Tounsi, Acta Phys. Pol. B27 (1996) 1035.

[17] P. Lévai, J. Zimányi, Phys. Lett. B304 (1993) 203.

[18] T.S. Biró, P. Lévai, B. Müller, Phys. Rev. D42 (1990) 3078. 
[19] X.-M. Xu, D. Kharzeev, H. Satz and X.-N. Wang, Phys. Rev. C53 (1996) 3051.

[20] V.V. Klimov, Zh. Eksp. Teor. Fiz. 82 (1982) 336 (Sov. Phys. JETP 55 (1982) 199);

H.A. Weldon, Phys. Rev. D26 (1982) 1394, D26 (1982) 2789;

K. Kajantie, P.V. Ruuskanen, Phys. Lett. B121 (1983) 352;

J.P. Blaizot, J.Y. Ollitrault, Phys. Rev. D48 (1993) 1390.

[21] E. Braaten, R.D. Pisarski, Nucl. Phys. B339 (1990) 310.

[22] F. Karsch, M.T. Mehr, and H. Satz, Z. Phys. C37 (1988) 617.

[23] A. Peshier, B. Kämpfer, O.P. Pavlenko, G. Soff, Phys. Rev. D54 (1996) 2399.

[24] A. Ukawa, Nucl. Phys. A498 (1989) 227c.

[25] J. Rafelski, B. Müller, Phys. Rev. Lett. 48 (1982) 1066, 56 (1986) 2334;

T.S. Biró, J. Zimányi, Phys. Lett. B113 (1982) 6., Nucl. Phys. A395 (1983) 525;

T. Matsui, B. Svetitsky, L.D. McLerran, Phys. Rev. D34 (1986) 783.

[26] M. Gyulassy, X.N. Wang, Nucl. Phys. B420 (1994) 583.

[27] J. Smith and R. Vogt, LBL-38282, ITP-SB-95-60, Z. Phys. C, in press. 


\begin{tabular}{|c|c|c|c|c|}
\hline \multicolumn{5}{|c|}{ RHIC } \\
\hline & \multicolumn{2}{|c|}{$m_{c}=1.2 \mathrm{GeV}$} & \multicolumn{2}{c|}{$m_{c}=1.5 \mathrm{GeV}$} \\
\hline & parton gas & minijet gas & parton gas & minijet gas \\
\hline$m=0 g$ & 3.2 & 0.053 & 0.93 & 0.0128 \\
\hline$m \neq 0 g$ & 4.6 & 0.065 & 1.27 & 0.0154 \\
\hline$m=0 g+q$ & 3.7 & 0.014 & 1.07 & 0.0027 \\
\hline$m \neq 0 g+q$ & 4.9 & 0.016 & 1.38 & 0.0030 \\
\hline
\end{tabular}

Table 1: The total thermal $c \bar{c}$ pair yield from a parton gas and a minijet gas at RHIC. We consider both a massless and massive pure gluon gas and a quark-gluon system with massless and massive components. 


\begin{tabular}{|c|c|c|c|c|}
\hline \multicolumn{5}{|c|}{ LHC } \\
\hline & \multicolumn{2}{|c|}{$m_{c}=1.2 \mathrm{GeV}$} & \multicolumn{2}{c|}{$m_{c}=1.5 \mathrm{GeV}$} \\
\hline & parton gas & minijet gas & parton gas & minijet gas \\
\hline$m=0 g$ & 102 & 39 & 43 & 21 \\
\hline$m \neq 0 g$ & 198 & 101 & 76 & 47 \\
\hline$m=0 g+q$ & 145 & 22 & 60 & 9.7 \\
\hline$m \neq 0 g+q$ & 245 & 38 & 94 & 15.6 \\
\hline
\end{tabular}

Table 2: The total thermal $c \bar{c}$ pair yield from a parton gas and a minijet gas at LHC. We consider both a massless and massive pure gluon gas and a quark-gluon system with massless and massive components. 


\section{Figure Captions}

Fig. 1: The time evolution of the plasma produced in $\mathrm{Au}+\mathrm{Au}$ collisions at RHIC is examined for a parton gas in (a), (c), (e) and (g) and a minijet gas in (b), (d), (f) and (h). The temperature is shown in (a) and (b), the energy density in (c) and (d), the square of the sound speed in (e) and (f) and the plasma volume in (g) and (h). The solid curve is for a massless gluon gas only while the dashed curve is for a gas of massive gluons. The dot-dashed curve is calculated assuming massless gluons and light quarks and $m_{s}=150 \mathrm{MeV}$ for the strange quark. The dotted curve is the result when the quarks and gluons have effective masses.

Fig. 2: The same as fig. 1 for $\mathrm{Pb}+\mathrm{Pb}$ collisions at LHC.

Fig. 3: Time dependence of the effective quark and gluon masses. The parton gas results are given in (a) RHIC and (b) LHC. The minijet gas results are shown in (c) RHIC and (d) LHC. The RHIC results are given for $\mathrm{Au}+\mathrm{Au}$ collisions, the LHC results for $\mathrm{Pb}+\mathrm{Pb}$ collisions. The solid curve is the effective light quark mass, the dashed is the strange quark mass. The dot-dashed curve is the effective gluon mass.

Fig. 4: The production rate (a), (c) and $c \bar{c}$ pair yield (b), (d) are given for $\mathrm{Au}+\mathrm{Au}$ collisions at RHIC with $m_{c}=1.2 \mathrm{GeV}$. The parton gas results are given in (a) and (b) while the minijet gas results are shown in (c) and (d). The solid curve is for a massless gluon gas only while the dashed curve is for a gas of massive gluons. The dot-dashed curve is calculated assuming massless gluons and light quarks and $m_{s}=150 \mathrm{MeV}$ for the strange quark. The dotted curve is the result when the quarks and gluons have effective masses.

Fig. 5: The same as in fig. 4 for $\mathrm{Au}+\mathrm{Au}$ collisions at RHIC with $m_{c}=1.5 \mathrm{GeV}$.

Fig. 6: The same as in fig. 4 for $\mathrm{Pb}+\mathrm{Pb}$ collisions at $\mathrm{LHC}$ with $m_{c}=1.2 \mathrm{GeV}$.

Fig. 7: The same as in fig. 4 for $\mathrm{Pb}+\mathrm{Pb}$ collisions at LHC with $m_{c}=1.5 \mathrm{GeV}$.

Fig. 8: The $c \bar{c}$ pair mass distribution from $\mathrm{Au}+\mathrm{Au}$ collisions at $\mathrm{RHIC}$ with $m_{c}=1.2$ $\mathrm{GeV}(\mathrm{a})$, (b) and $m_{c}=1.5 \mathrm{GeV}(\mathrm{c}),(\mathrm{d})$. The parton gas results are given in (a) and (c) while the minijet gas results are shown in (b) and (d). The solid curve is for a massless gluon gas only while the dashed curve is for a gas of massive gluons. The dot-dashed curve is calculated assuming massless gluons and light quarks and $m_{s}=150 \mathrm{MeV}$ for the strange quark. The dotted curve is the result when the quarks and gluons have effective masses.

Fig. 9: The same as in fig. 8 for $\mathrm{Pb}+\mathrm{Pb}$ collisions at LHC. 

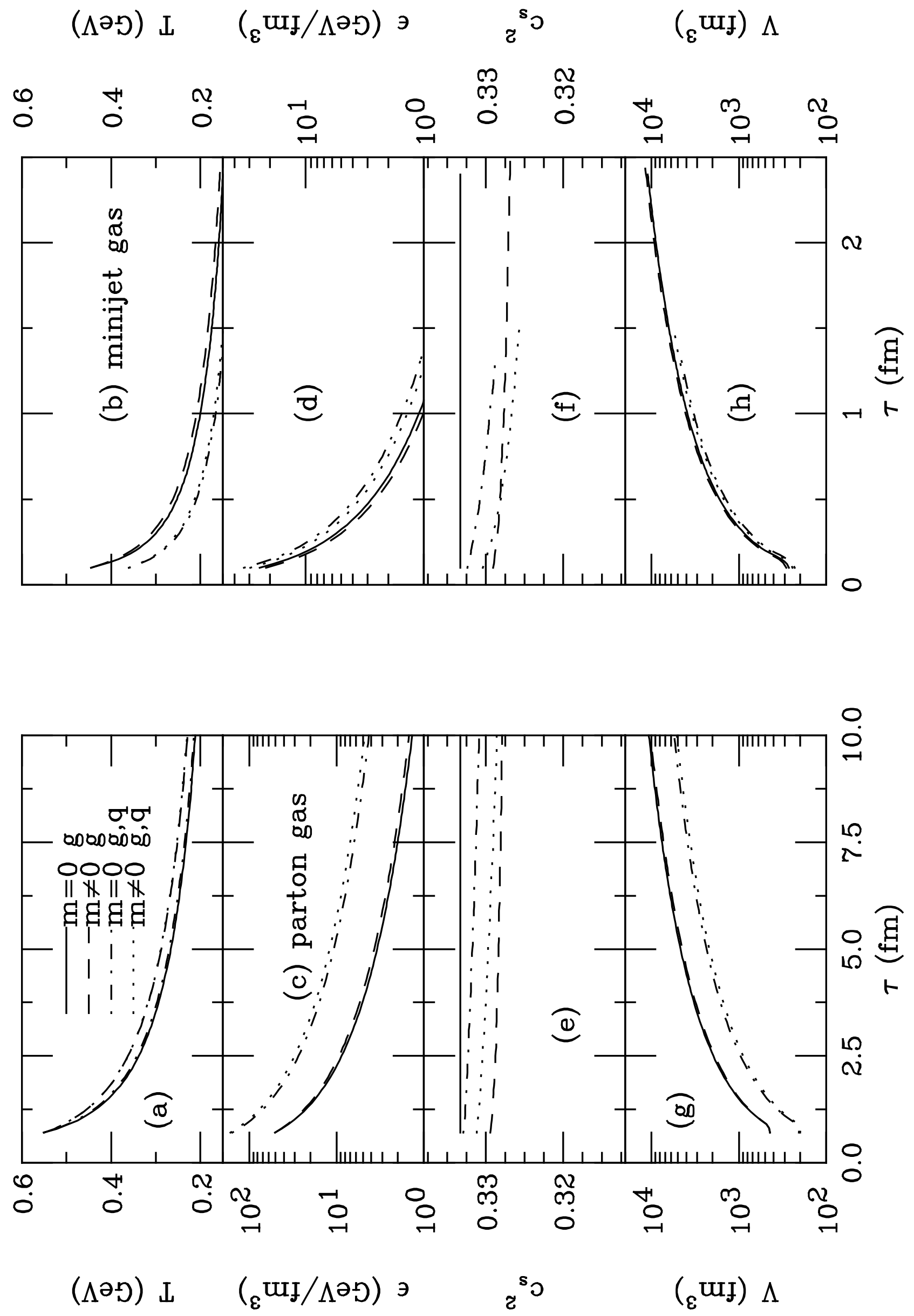

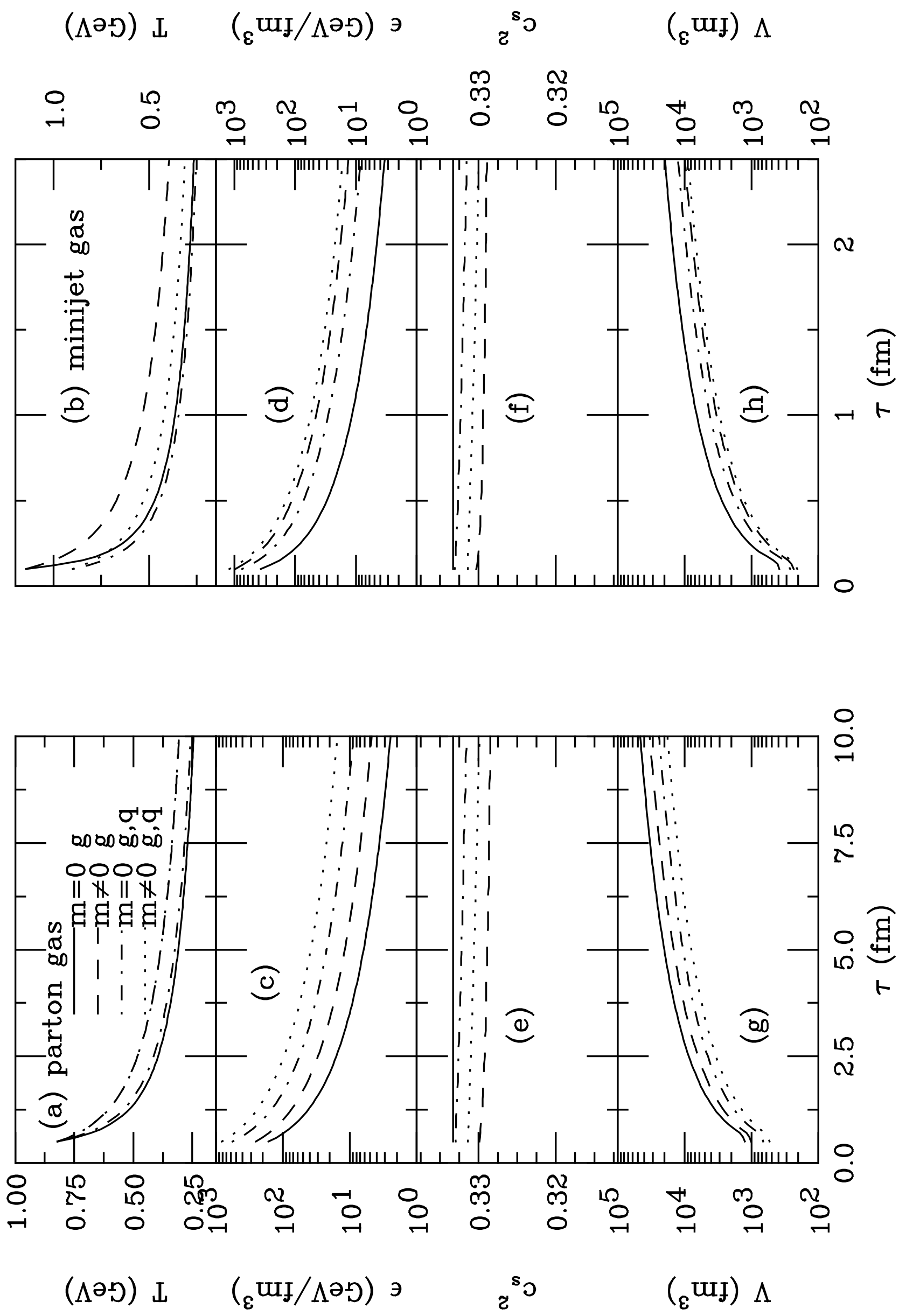


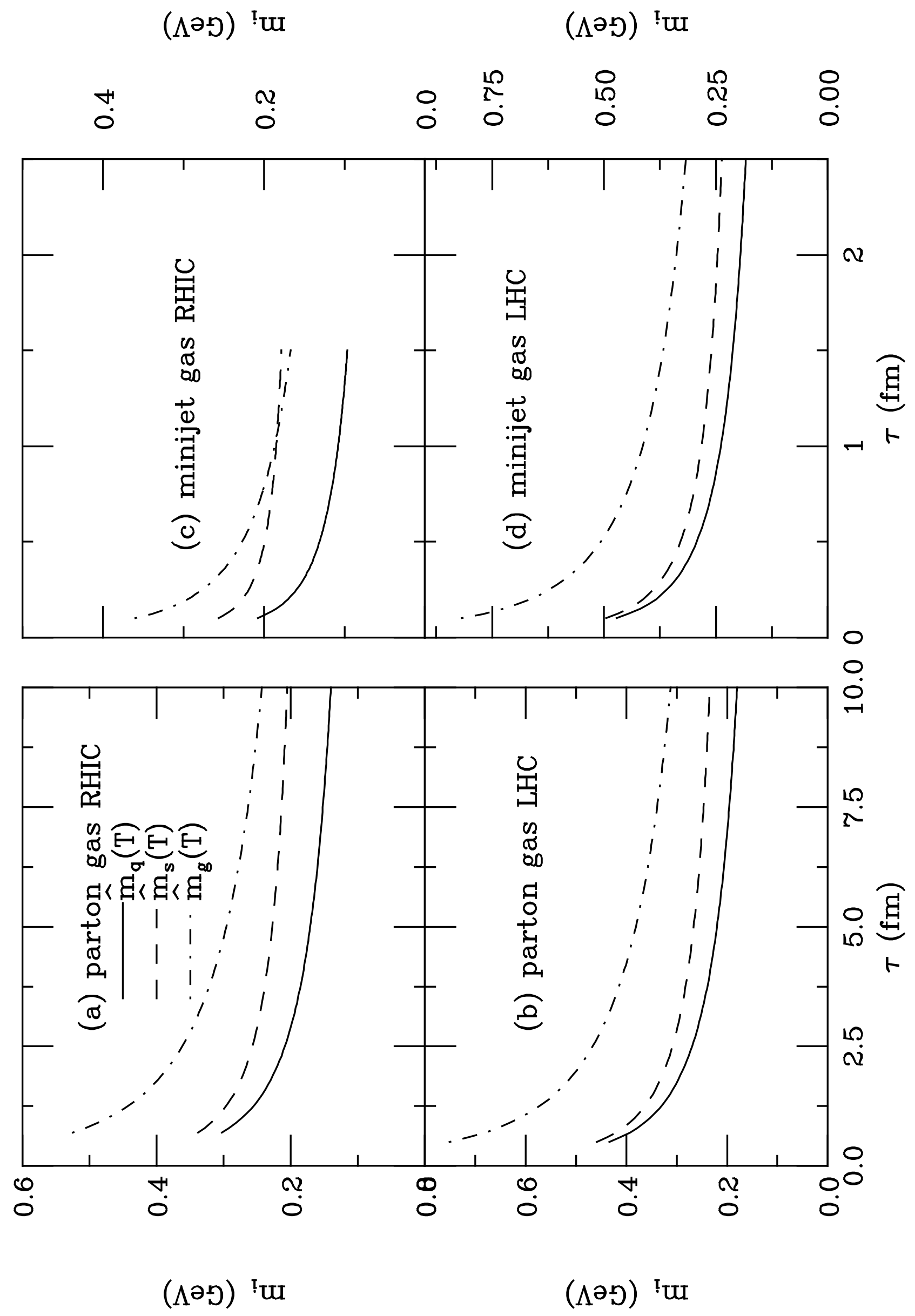




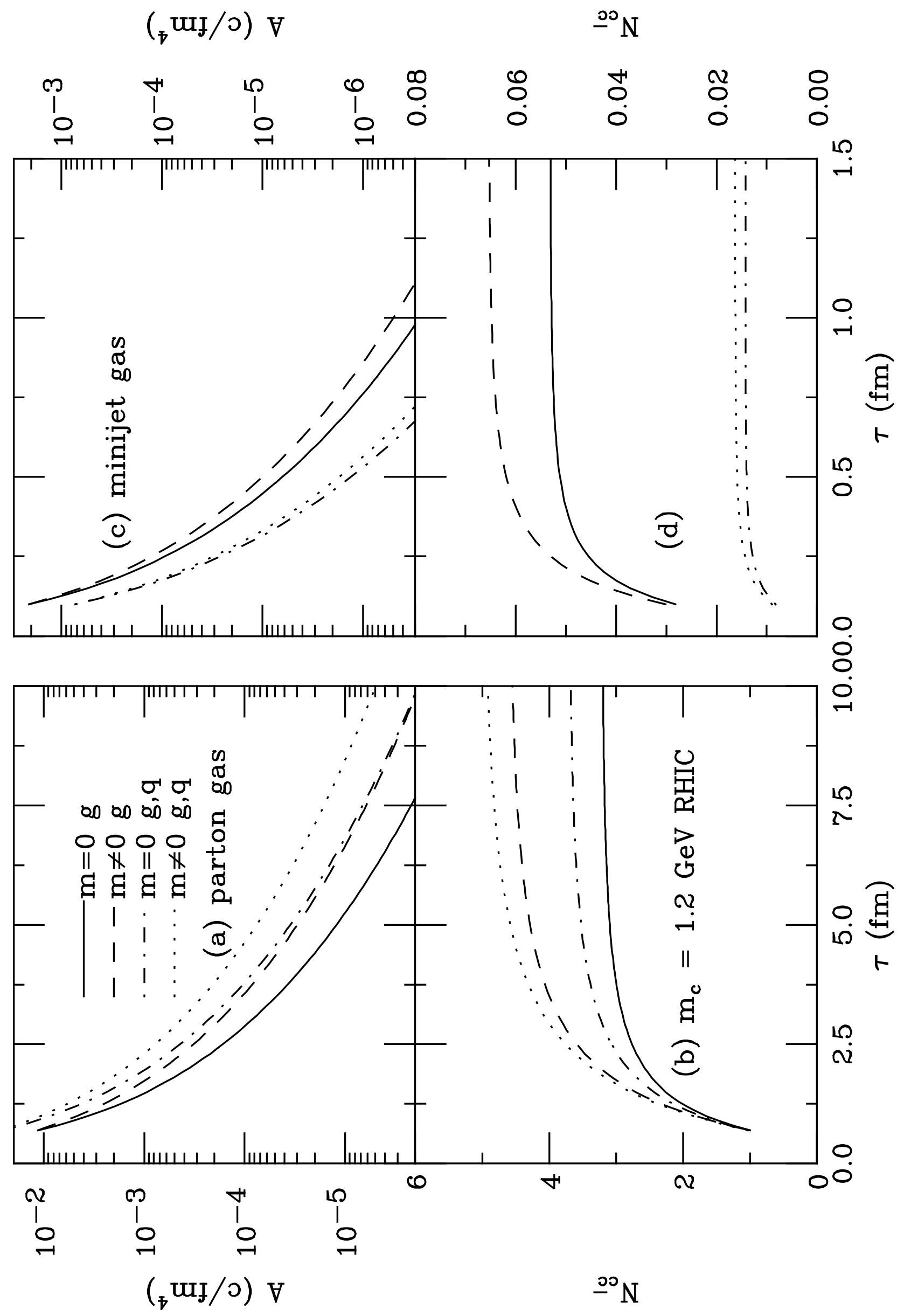




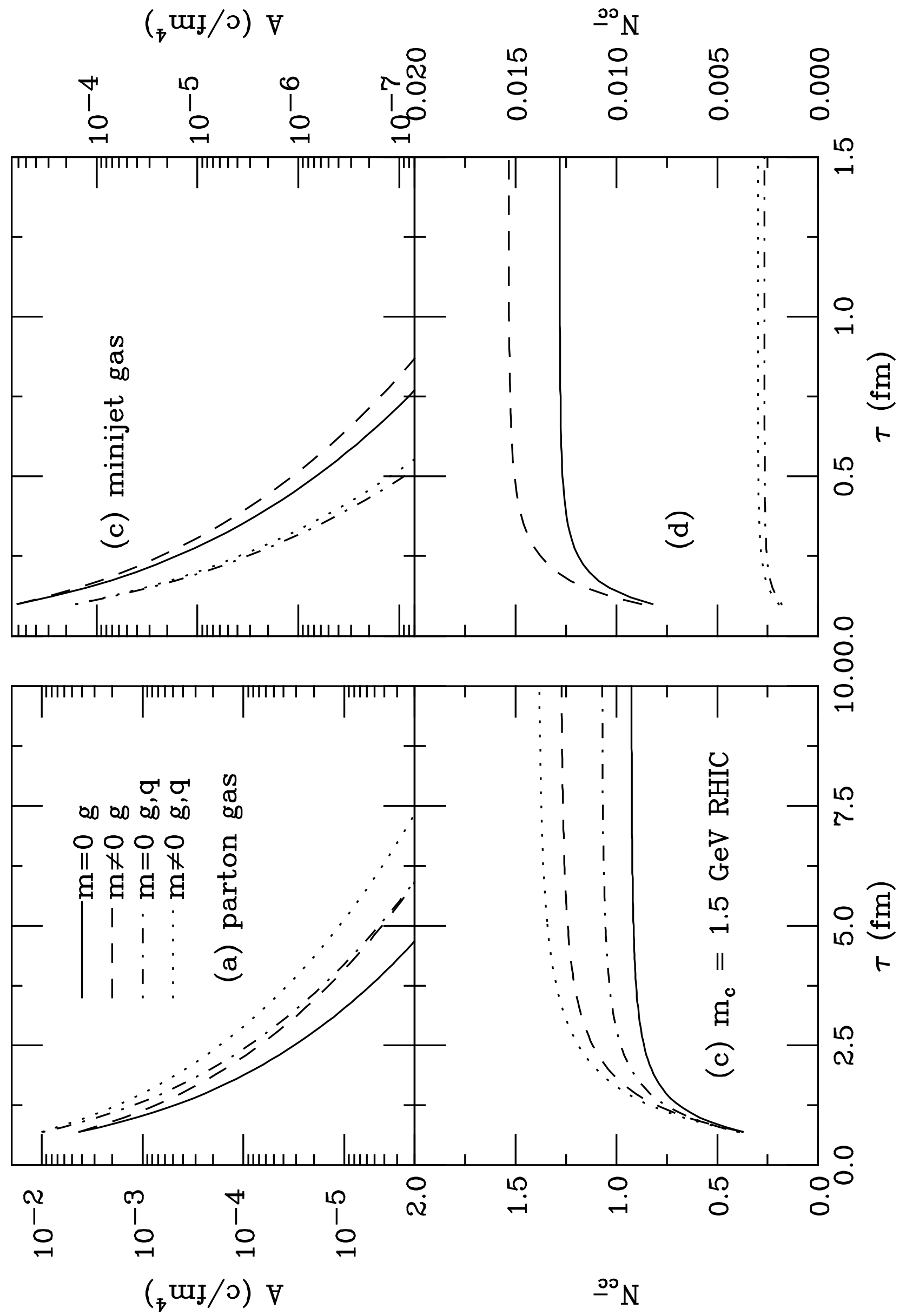




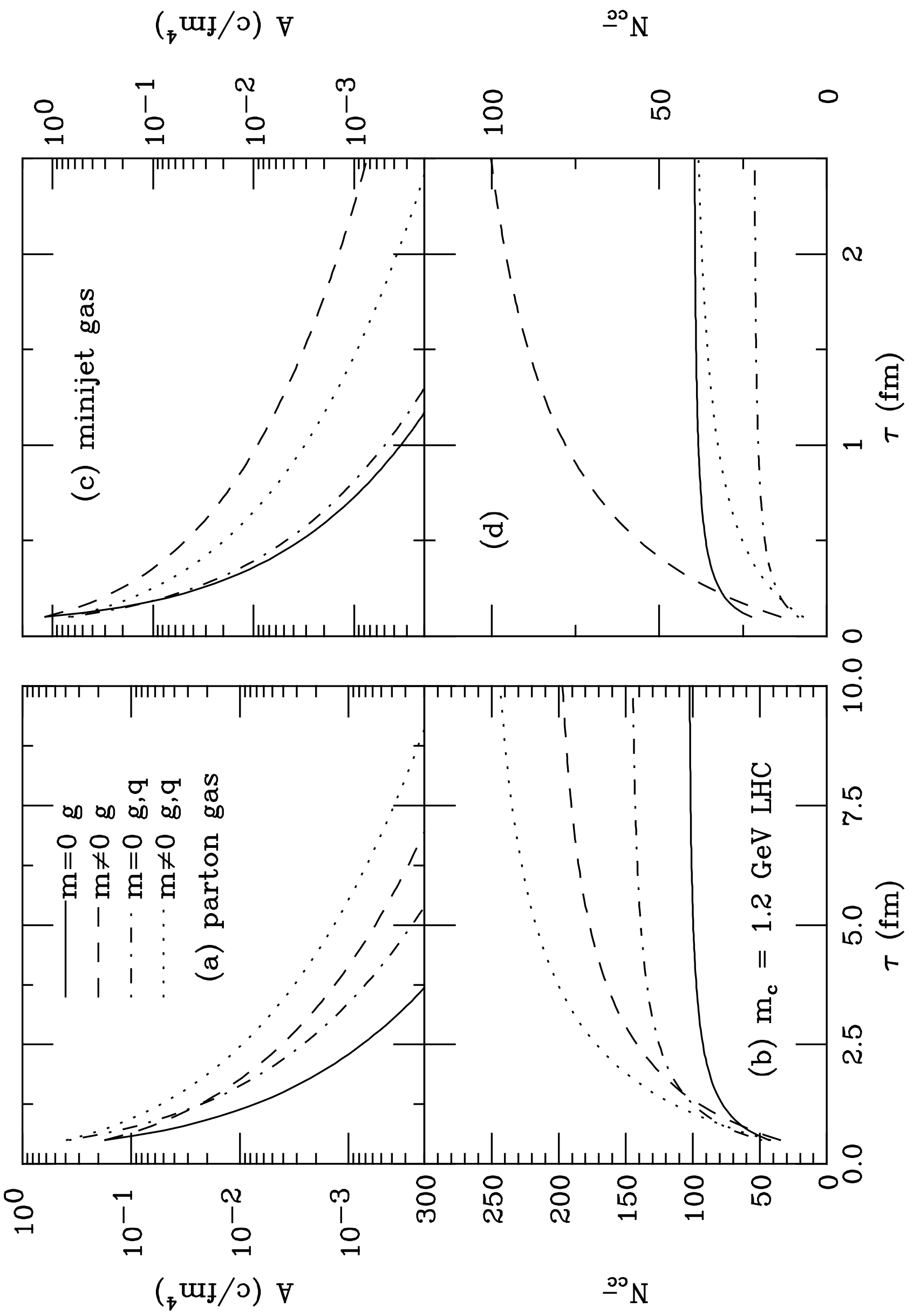



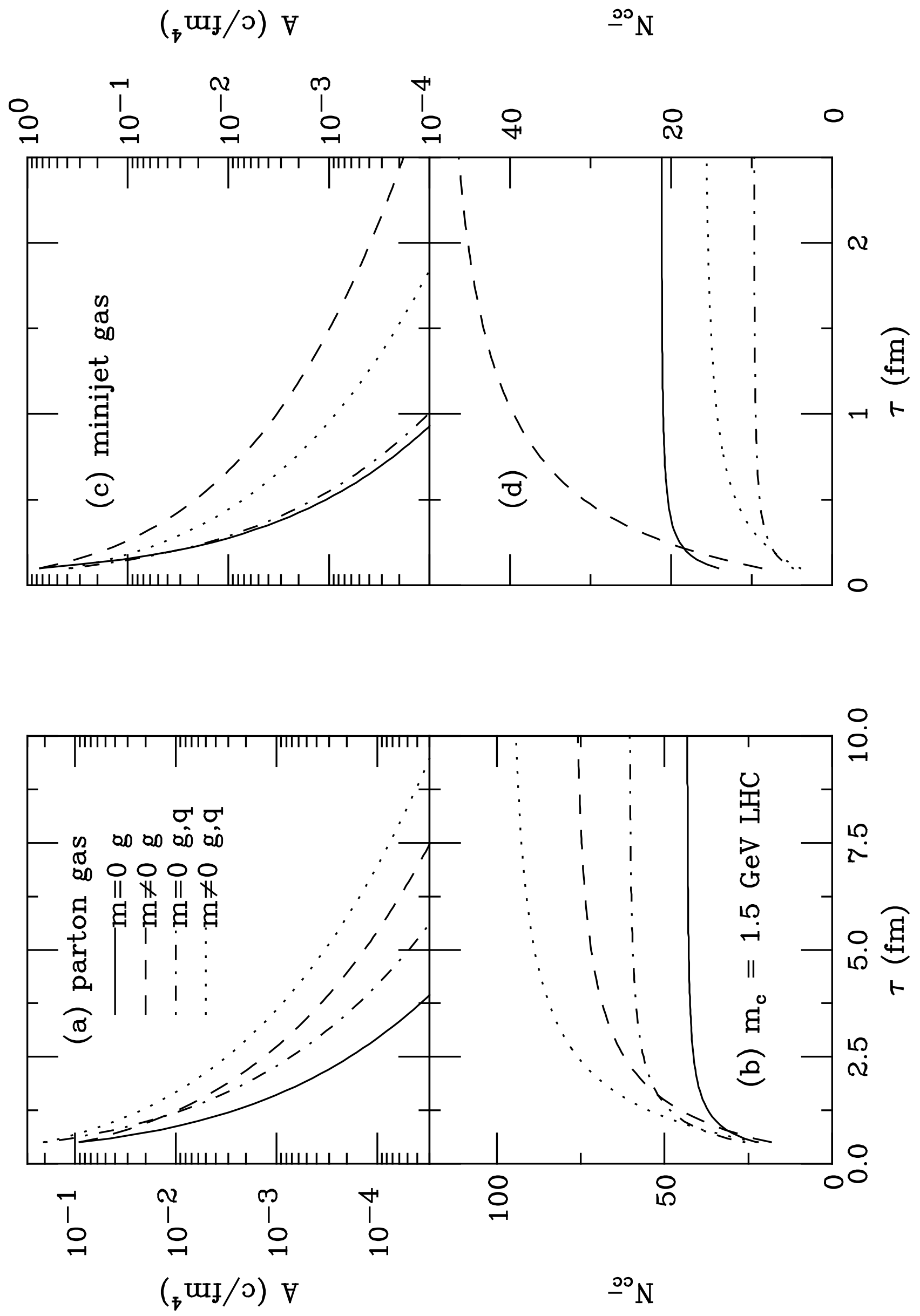

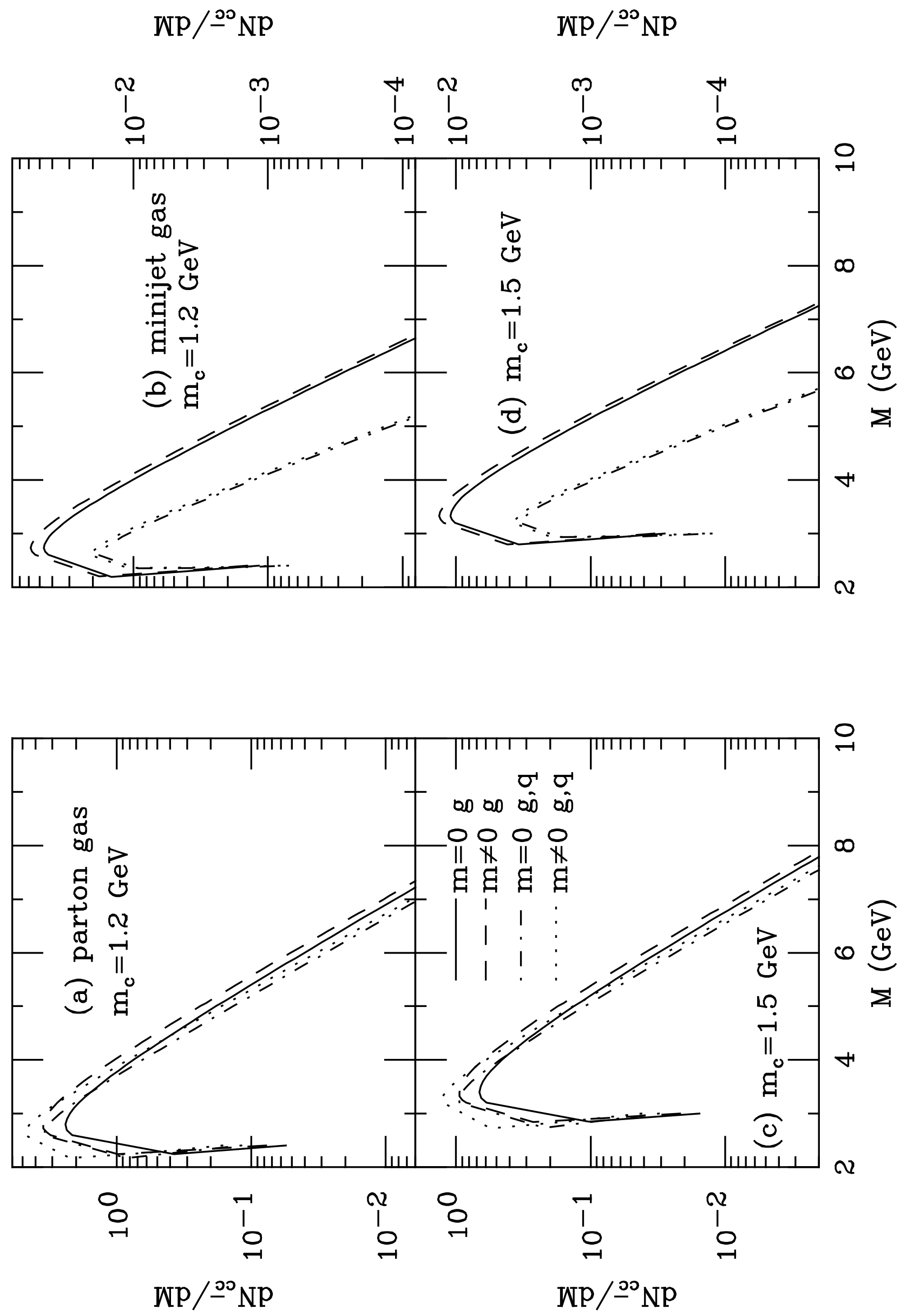

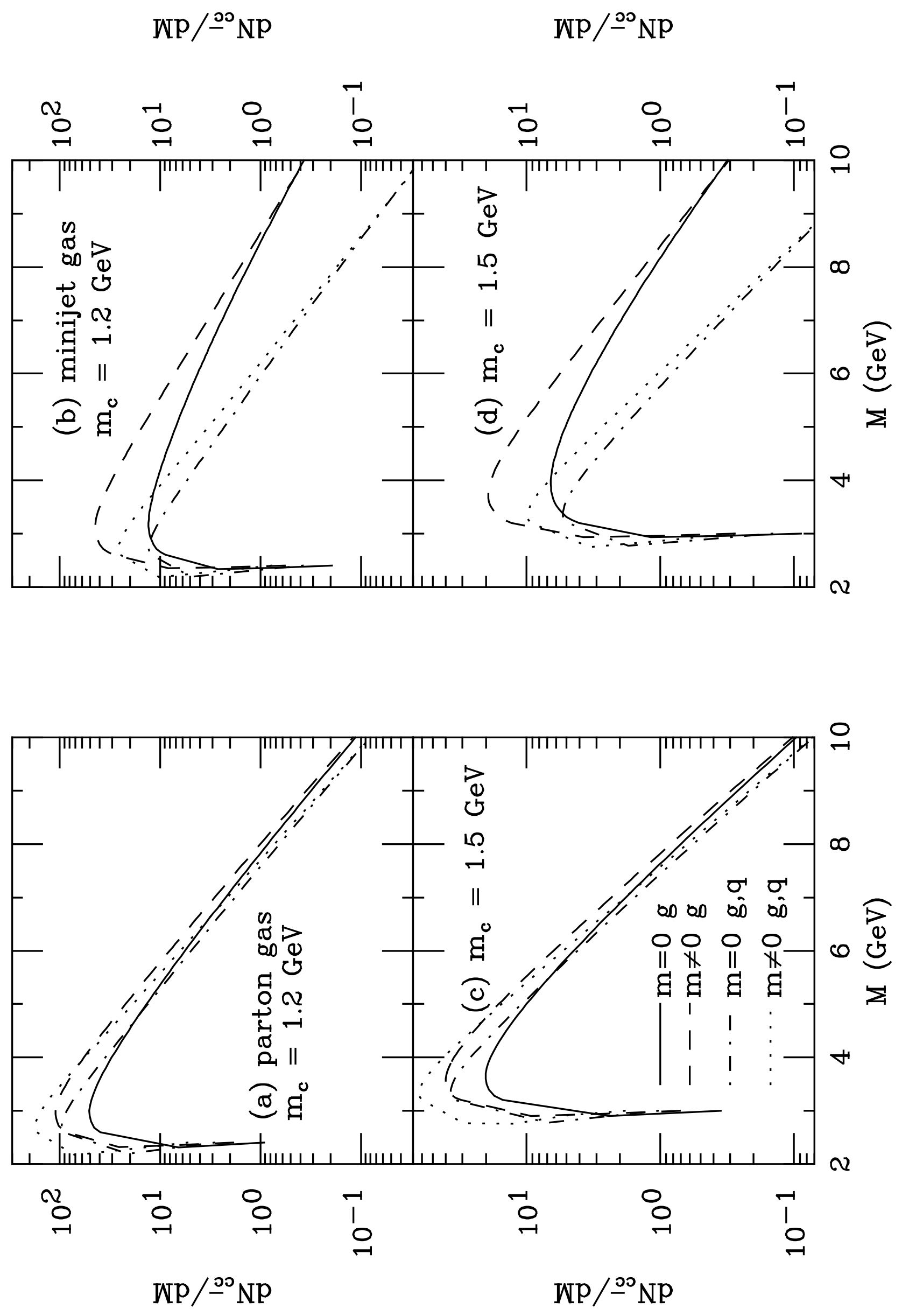